\title{
How to Distinguish Dementia with Lewy Bodies from Alzheimer Disease?
}

\author{
Pietro Tiraboschi ${ }^{*}, 1,2$ and Ugo P. Guerra ${ }^{3}$ \\ ${ }^{I}$ Department of Neurology, Ospedale Niguarda Ca' Granda, Milano, Italy \\ ${ }^{2}$ Fondazione Europea Ricerca Biomedica, Centro Alzheimer Ospedale Briolini di Gazzaniga, Bergamo, Italy \\ ${ }^{3}$ Department of Nuclear Medicine, Ospedali Riuniti di Bergamo, Bergamo, Italy
}

\begin{abstract}
Dementia with Lewy (DLB) bodies is the most common type of degenerative dementia after Alzheimer Disease (AD). Although an accurate diagnosis of DLB is important for adequate prognosis and therapy, its differentiation from other dementias and, especially, from AD may be extremely challenging for clinicians, as highlighted by the high variability in reported sensitivity $(0.22-0.83)$ and specificity $(0.79-1.00)$ rates for a diagnosis of probable DLB applying current clinical criteria. Various kinds of imaging procedures, including conventional MRI and brain perfusion SPECT, have been proposed for improving diagnostic accuracy, especially for most controversial cases. Among such techiques, those using radioactive tracers measuring the striatal binding at pre-synaptic dopamine transporter sites or myocardial uptake in post-ganglionic sympathetic fibers have emerged as the most useful for diagnostic purposes.
\end{abstract}

Keywords: Alzheimer disease, Dementia with Lewy bodies, DAT scan, MIBG myocardial scintigraphy.

\section{DEMENTIA WITH LEWY BODIES: CLINICAL PHENOMENOLOGY AND DIAGNOSTIC CONTRO- VERSIES}

Dementia with Lewy bodies (DLB) has been reported to be the second most common form of degenerative dementia, after Alzheimer Disease (AD). The importance of identifying this entity lies essentially in its pharmacologic management, with a potential good response to cholinesterase inhibitors [1], but increased sensitivity to adverse effects of neuroleptic drugs $[2,3]$.

Much attention has been focused on the identification of reliable criteria that may help the clinician to discriminate DLB from other dementias, and especially from AD [4]. In addition to cognitive decline, the core clinical features of DLB, according to the Consortium on DLB criteria [4, 5], are visual hallucinations $(\mathrm{VH})$, which typically are recurrent, well formed and detailed, fluctuating cognition with pronounced variations in attention and alertness, and spontaneous (i.e., not drug-induced) features of parkinsonism, with an overrepresentation of the "postural instability-gait difficulty" phenotype [6]. The motor manifestations of parkinsonism may also include limb rigidity, bradykinesia, and a symmetrical postural tremor, while unilateral rest tremor is relatively uncommon. Neuropsychologically, compared to AD, patients with DLB tend to show a different pattern of cognitive impairment, with more preserved memory [7], but worse performances on attentional and executive tasks $[8,9]$ and on tests of visuospatial/constructional abilities $[10,11]$. However, despite emphasis placed on these distinctive characteristics, while neuropathologic series have demonstrated high

*Address correspondence to this author at the Department of Neurology Ospedale Niguarda Ca' Granda, Milano, Italy; Tel: +39 02 6444.2137; Fax: +390264442819; E-mail: ptiraboschi@yahoo.com accuracy for the clinical diagnosis of $\mathrm{AD}$ [12], the accuracy for the clinical diagnosis of DLB using the criteria originally suggested by the Consortium on DLB [4] has been in general less satisfactory [13-17], essentially because some of the "core" clinical features of DLB may not invariably appear even during the entire course of the disease [18] or may overlap with some extent with AD [19]. The presence and severity of concurrent AD pathology in DLB modifies the clinical presentation, with decreased rates of $\mathrm{VH}$ and parkinsonism as neurofibrillary tangle pathology increases, making these cases harder to be recognized [19]. Another reason for "missing" DLB clinically, which clearly emerged from the above mentioned studies [13-17], was the authors' failure to reliably identify fluctuations, a symptom which even today remains hard to define, identify, and reliably assess, despite recent proposals of structured methods for its detection $[20,21]$. As a result, using Consensus clinical criteria as originally formulated [4], DLB often goes unrecognized and mostly misdiagnosed as AD.

To obviate this, diagnostic criteria for DLB have recently been updated [5] and, although it was still acknowledged the importance of $\mathrm{VH}$, fluctuations in attention and vigilance, and spontaneous parkinsonism as "core" features of the disease, a substantial weight has also been given to REM behaviour disorder (RBD), neuroleptic hypersensitivity, and decreased striatal binding at pre-synaptic dopamine transporter sites [22-24], so that the presence of at least one of these features with just one "core" symptom/sign is now considered sufficient to warrant a diagnosis of probable DLB. It should be highlighted, however, that at least two of the "suggestive" features (neuroleptic hypersensitivity and decreased striatal binding of radioactive tracers) would not be useful to corroborate a hypothetical diagnosis of DLB (shift from the possible to the probable category) if parkinsonism is the only "core" feature exhibited by the patient. In this case, in fact, reduced striatal binding and 
exacerbation of motor signs following antipsychotic treatment are largely expected (circularity). It is also worth mentioning that, although decreased striatal binding of radioactive tracers has been proven to have good sensitivity and specificity to DLB [22-24], and severe nigrostriatal degeneration, as indicated by abnormal (low binding) dopamine transporter activity on SPECT or PET imaging, is considered the most reliable biological diagnostic marker for this condition, there are clinical and pathologic studies [25, 26] where this finding has also been reported for patients with AD. Fig. (1) shows examples of a normal dopamine transporter scan (a) and of a patient affected with DLB (b).
For these reasons, alternative methods to support the diagnosis of DLB have been proposed, such as myocardial scintigraphy with metaiodobenzyl guanidine (MIBG), an analogue of norepinephrine whose decreased uptake reflects the damage of post-ganglionic sympathetic cardiac innervation [27-29]. It is well known that severe autonomic dysfunction causes many symptoms, including orthostatic hypotension, that is more frequently seen in synucleinopathies [multiple system atrophy (MSA) $>$ DLB $>$ idiopathic Parkinson disease (PD)] than in AD or frontotemporal dementias. MIBG uptake has been found to be particularly decreased in DLB patients with orthostatic

(a)

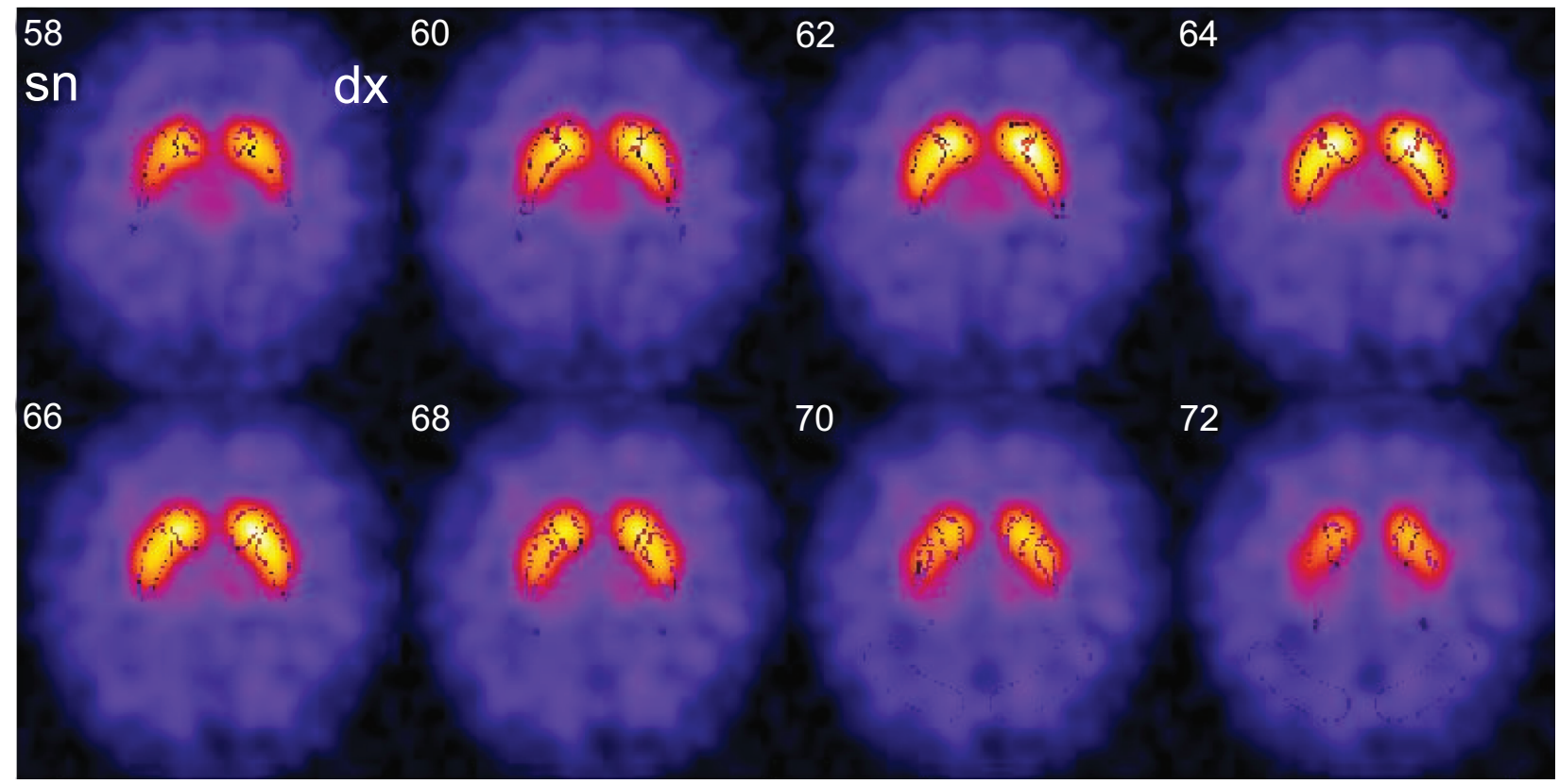

(b)

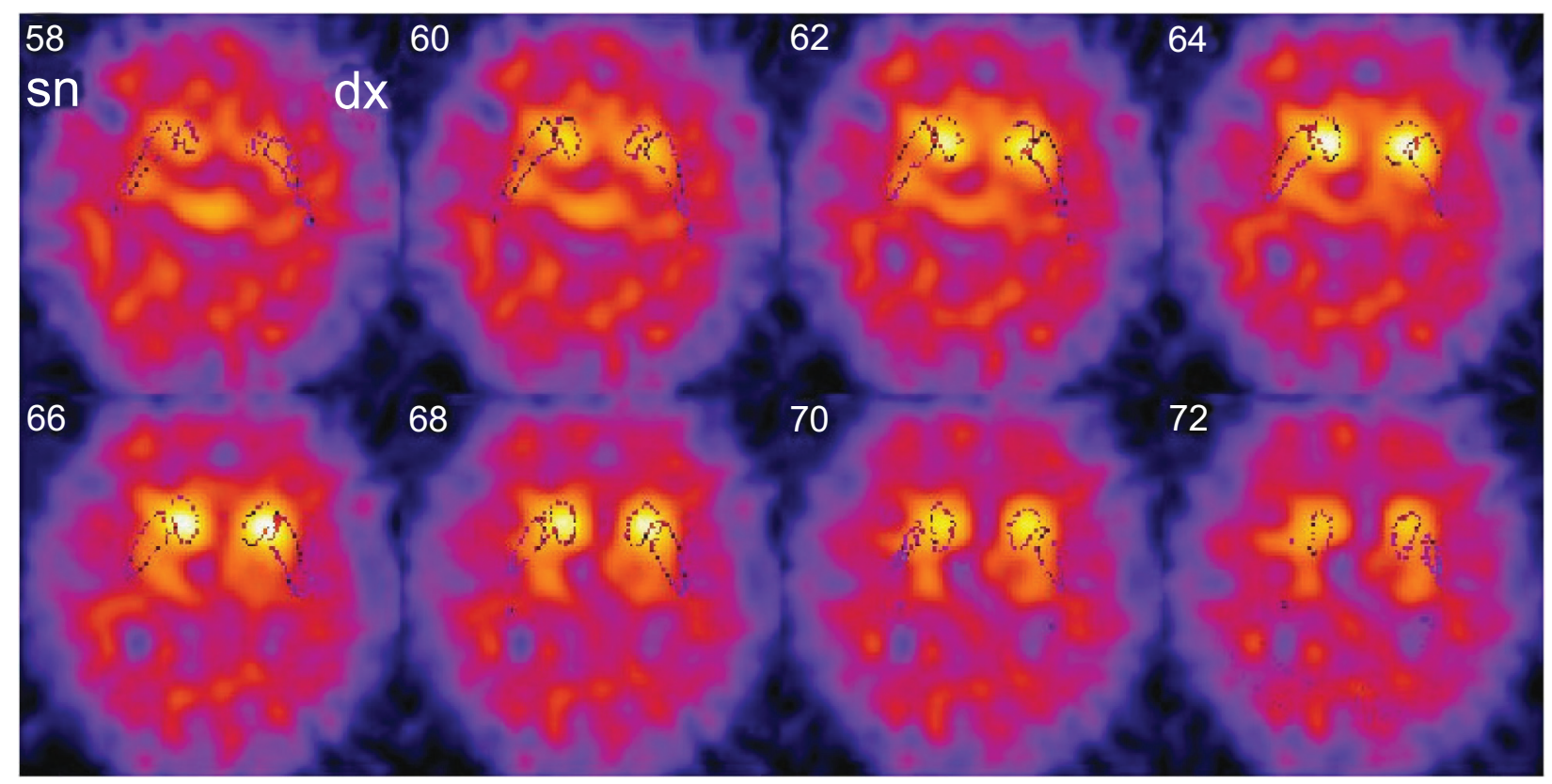

Fig. (1). Transverse reconstruction ${ }^{123}$ I-FPCIT SPECT scan in Alzheimer dementia (a) and in Lewy body dementia (b). Black dots are automatically drawn according to software BasGan [52]. 
hypotension [30]. However, as a reduction in tracer binding at pre-synaptic dopamine transporter sites may reflect a level of nigrostriatal degeneration still insufficient to determine clinical parkinsonism, so reduced myocardial MIBG uptake may reflect pathologic changes in post-ganglionic sympathetic cardiac innervation still insufficient to determine orthostatic hypotension. Therefore, for the high sensitivity to pathologic changes caused by DLB (the presence of Lewy neurites has been described in the cardiac plexus and in post-ganglionic sympathetic cardiac terminals) even before clinical expression, MIBG scintigraphy could indeed overcome the difficulties of clinical criteria alone to identify cases with DLB. Decreased MIBG uptake on myocardial scintigraphy has also been reported to have excellent specificity to DLB (and PD). A normal uptake is, in fact, expected, in MSA (where structural changes in the autonomic system are essentially restricted to pre-ganglionic sympathetic innervation), in $\mathrm{AD}$ (where autonomic dysfunction is rare and, when present, is generally attributed to structural changes in the brainstem and/or hypothalamus) [31], and in frontotemporal dementia [32]. MIBG scintigraphy (examples in Fig. 2a, b) may be preferred to dopamine transporter (DAT) imaging because it is considerably less expensive and its sensitivity and specificity to DLB is completely independent of the presence of parkinsonism [31]. On the other hand, compared to DATimaging, MIBG scintigraphy has some disadvantages, such as the fact that several common illnesses in the elderly (including heart infarct, heart failure, dilatative cardiomyopathy, and dysautonomic diabetic neuropathy) may interfere with MIBG uptake. This can lead to false positive results in patients suffering from dementias other than DLB. In this respect, it should be highlighted that the complete $(100 \%)$ sensitivity and specificity of MIBG scintigraphy to DLB recently reported by some investigators [31] has been obtained in a highly selected sample, because all patients with medical conditions potentially interfering with MIBG uptake were excluded from the study.

Another issue is the lack of standardization with regard to timing, acquisition, and processing of images. Although planar acquisitions are used by all investigators [27-37], acquisition times after MIBG injection are extremely variable (ranging from 15 to 120 minutes for early images and 200 to 240 minutes for delayed images) and heart-tomediastinum ratios are determined on regions of interest that differ in shape and size, explaining the cut-off variability reported in the literature (1.5-2.6 for late images) [27-37].

\section{Other Laboratory and Neuroimaging Investigations}

In DLB, the standard EEG may show early slowing, epoch-by-epoch fluctuation, and transient slow-wave activity [38]. EEG variability, assessed by mean frequency analysis and compressed spectral arrays (CSA), may be particularly useful in discriminating between DLB from AD since the earliest stages of dementia. Specifically, particular frequency bands $(5.6-7.9 \mathrm{~Hz})$ seem to be peculiar to DLB and appear to be related to the presence and severity of cognitive fluctuations [39]. As of yet, there are no clinically applicable genotypic or CSF markers to support a diagnosis of DLB, although phosphorilated and total tau are in general lower in DLB than AD [40, 41]. Conversely, Abeta 42 is usually comparable, reflecting high plaque deposition in both DLB and $\mathrm{AD}$ [42]. On coronal MRI, hippocampal and medial temporal lobe atrophy is less pronounced in DLB than AD [43]. This is in keeping with autopsy findings but, in earlystage dementia, preservation of medial temporal lobe volumes may be seen in both diseases. Occipital hypoperfusion/hypometabolism on SPECT [44] or PET [45] are changes usually associated to DLB, but not to AD. However, these abnormalities are seen in only approximately $50-70 \%$ of DLB cases (high specificity, but somewhat poor sensitivity). Of note, in two recent studies comparing brain perfusion SPECT and MIBG myocardial scintigraphy, the latter was significantly superior in the identification of DLB subjects $[46,47]$.

(a)

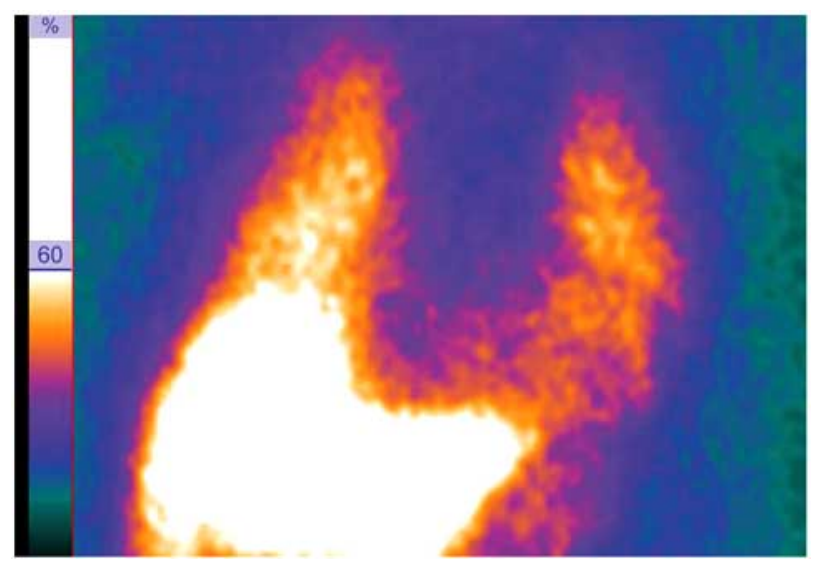

(b)

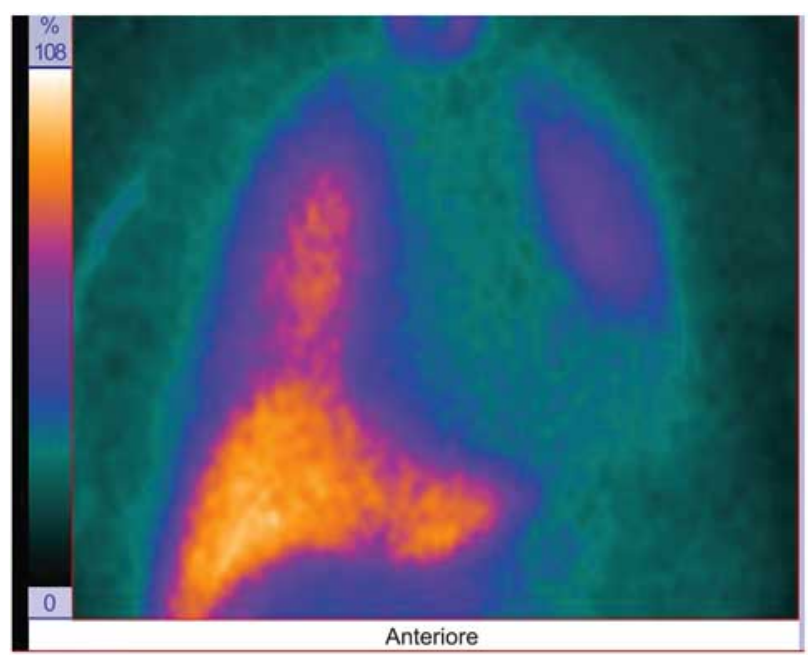

Fig. (2). Planar scintigraphy with ${ }^{123} \mathrm{I}-\mathrm{MIBG}$ in Alzheimer dementia (a) and in Lewy body dementia (b).

\section{CONCLUSION}

DLB may present as a primary neuropsychiatric syndrome characterized by cognitive decline, in which case diagnostic differentiation is from other causes of dementia, particularly $\mathrm{AD}$, or it may develop later, in a patient already labelled as having PD. The latter presentation poses considerably less challenge to the clinician's diagnostic 
ability since, in AD, extrapyramidal signs (EPS) are variable and, if present, usually follow the onset of cognitive deterioration. Such an order of presentation of cognitive and motor features is also typical of DLB, making its distinction from AD with extrapyramidal signs extremely hard.

There is evidence that the three core features of DLB (VH, fluctuations, and parkinsonism) do not have the same diagnostic weight. For example, in a recent neuropathologic series of patients with mild dementia [48], while $\mathrm{VH}$ at presentation were a strong predictor of DLB at autopsy, the presence of parkinsonism did not appear to enhance diagnostic accuracy. In fact, EPS were exhibited by only approximately one fourth of the DLB cases and, above all, there were no significant differences in its prevalence between the cases with DLB and those with AD $(26 \% v s$ $16 \%$ ). An important implication of this study is that, in earlystage dementia, several patients clinically labeled as DLB on the basis of the presence of parkinsonism alone have a high risk to be misdiagnosed (false positivity). It is especially in these cases (labeled as possible DLB on the basis of the presence of a single core feature) that ancillary investigations, such as neuroimaging, might be most important in enhancing accuracy of diagnosis.

With one exception [49], clinical-pathologic correlations with the original DLB criteria [4] have not been very good [13-17]. Specifically, most of validation studies [13-17] have shown good specificity, but only modest sensitivity, for the clinical diagnosis of probable DLB. That is, applying the Consensus criteria as originally formulated, the presence of two or more core features (clinically, probable DLB) was strongly predictive of DLB at autopsy but, unfortunately, few patients with DLB at autopsy showed more than one core feature during the entire course of the disease. As a result, DLB was underestimated and mostly misdiagnosed as AD. It is likely that the new criteria [5] might improve this situation. In support of this, is a recent validation study [50] which, using the revised criteria and placing particular emphasis on RBD, has shown not only excellent specificity, but also high sensitivity (87\%) for the diagnosis of probable DLB. There is also preliminary evidence of the potentially important role of ancillary investigations in improving diagnostic accuracy in diagnostically uncertain cases, as indicated by a recent study of patients with only possible DLB, where an abnormal DAT-scan at baseline strongly predicted the appropriateness of this diagnosis, preceding the appearance of other distinctive clinical features [51].

\section{REFERENCES}

[1] McKeith I, Del Ser T, Spano PF, et al. Efficacy of rivastigine in dementia with Lewy bodies: a randomized, double-blind, placebocontrolled international study. Lancet 2000; 356: 2031-6.

[2] McKeith I, Fairbairn A, Perry R, Thompson P, Perry E. Neuroleptic sensitivity in patients with senile dementia of Lewy body type. BMJ 1992; 305: 673-8.

[3] Ballard C, Grace J, McKeith I, Holmes C. Neuroleptic sensitivity in dementia with Lewy bodies and Alzheimer's disease. Lancet 1998; 351: 1032-3.

[4] McKeith IG, Galasko D, Kosaka K, et al. Consensus guidelines for the clinical and pathologic diagnosis of dementia with Lewy bodies (DLB): report of the Consortium on DLB international workshop. Neurology 1996; 47: 1113-24.

[5] McKeith I, Dickson D, Lowe J, et al. Diagnosis and management of dementia with Lewy dodies: third report of the DLB consortium. Neurology 2005; 65: 1863-72.
[6] Jankovic J, McDermott M, Carter J, et al. Variable expression of Parkinson's disease: a base-line analysis of the DATATOP cohort. The Parkinson Study Group. Neurology 1990; 40: 1529-34.

[7] Connor DJ, Salmon DP, Sandy TJ, Galasko D, Hansen LA, Thal LJ. Cognitive profiles of autopsy-confirmed Lewy body variant $v s$ pure Alzheimer disease. Arch Neurol 1998; 55: 994-1000.

[8] Hansen LA, Salmon DP, Galasko D, et al. The Lewy body variant of Alzheimer's disease: a clinical and pathological entity. Neurology 1990; 40: 1-8.

[9] Ballard C, O’Brien J, Gray A, et al. Attention and fluctuating attention in patients with dementia with Lewy bodies and Alzheimer's disease. Arch Neurol 2001; 58: 997-82.

[10] Salmon DP, Galasko D, Hansen LA, et al. Neuropsychological deficits associated with diffuse Lewy body disease. Brain Cogn 1996; 31: 148-65.

[11] Ala TA, Hughes LF, Kyrouac GA, Ghobrial MW, Elble RJ. Pentagon copying is more impaired in dementia with Lewy bodies than in Alzheimer's disease. J Neurol Neurosurg Psychiatry 2001; 70: 483-8.

[12] Kosunen O, Soininen H, Paljarvi L, Heinonen O, Talasniemi S, Riekkinen PJ. Diagnostic accuracy of Alzheimer's disease. A neuropathologic study. Acta Neuropathol (Berl) 1996; 91: 185-93.

[13] Litvan I, MacIntyre A, Goetz CG, et al. Accuracy of the clinical diagnoses of Lewy body disease, Parkinson disease, and dementia with Lewy bodies: a clinicopathologic study. Arch Neurol 1998; 55: 969-78.

[14] Lopez OL, Litvan I, Catt KE, et al. Accuracy of four clinical diagnostic criteria for the diagnosis of neurodegenerative dementias. Neurology 1999; 53: 1292-9.

[15] Luis CA, Barker WW, Gajaraj K, et al. Sensitivity and specificity of three clinical criteria for dementia with Lewy bodies in an autopsy-verified sample. Int J Geriatr Psychiatry 1999; 14: 526-33.

[16] Verghese J, Crystal HA, Dickson DW, Lipton RB. Validity of clinical criteria for the diagnosis of dementia with Lewy bodies. Neurology 1999; 53: 1974-82.

[17] Hohl U, Tiraboschi P, Hansen LA, Thal LJ, Corey-Bloom J. Diagnostic accuracy of dementia with Lewy bodies. Arch Neurol 2000; 57: 47-51.

[18] Merdes AR, Hansen LA, Jeste DV, et al. Influence of Alzheimer pathology on clinical diagnostic accuracy in dementia with Lewy bodies. Neurology 2003; 60: 1586-90.

[19] Walker Z, Jaros E, Walker RWH, et al. Dementia with Lewy bodies: a comparison of clinical diagnosis, FP-CIT SPECT imaging and autopsy. J Neurol Neurosurg Psychiatry 2007; 78: 1176-81.

[20] Walker MP, Ayre GA, Cummings JL, et al. The clinician assessment of fluctuations and the one day assessment scale. Br J Psychiatry 2000; 177: 252-6.

[21] Ferman TJ, Smith GE, Boeve BF, et al. DLB fluctuations. Specific features that reliably differentiate DLB from AD and normal aging. Neurology 2004; 62: 181-7.

[22] Walker Z, Costa DC, Walker RWH et al., Differentiation of dementia with Lewy bodies from Alzheimer's disease using a dopaminergic presynaptic ligand, J Neurol Neurosurg Psychiatry 2002; 73: 134-40.

[23] O'Brien JT, Colloby S, Fenwick J, et al. Dopamine transporter loss visualized with FP-CIT SPECT in the differential diagnosis of dementia with Lewy bodies, Arch Neurol 2004; 61: 919-25.

[24] McKeith I, O'Brien JT, Walker Z, et al. Sensitivity and specificity of dopamine transporter imaging with ${ }^{123}$ I-FP-CIT-SPECT in dementia with Lewy bodies: a phase III, multicentre study. Lancet Neurol 2007; 6: 305-13.

[25] Rinne JO, Sahlberg N, Ruottinen H, Nagren K, Lehikoinen P. Striatal uptake of the dopamine reuptake ligand $[11 \mathrm{C}]$ beta-CFT is reduced in Alzheimer's disease assessed by positron emission tomography. Neurology 1998; 50: 152-6.

[26] Murray AM, Weihmueller FB, Marshall JF, Hurtig HI, Gottleib GL, Joyce JN. Damage to dopamine systems differs between Parkinson's disease and Alzheimer's disease with parkinsonism. Ann Neurol 1995; 37: 300-12.

[27] Yoshita M, Taki J, Yamada M. A clinical role for ${ }^{123}$ I-MIBG myocardial scintigraphy in the distinction between dementia of the Alzheimer's-type and dementia with Lewy bodies. J Neurol Neurosurg Psychiatry 2001; 71: 583-8.

[28] Oka H, Morita M, Onouchi K, Yoshioka M, Mochio S, Inoue K. Cardiovascular autonomic dysfunction in dementia with Lewy bodies and Parkinson disease. J Neurol Sci 2007; 254: 72-7. 
[29] Nakajima K, Yoshita M, Matsuo S, Taki J, Kinuya S. Iodine-123MIBG sympathetic imaging in Lewy-body diseases and related movement disorders. Quart J Nucl Med Mol Imaging 2008; 52: 378-87.

[30] Kobayashi S, Tatenu M, Morii H, Utsumi K, Saito T. Decreased cardiac MIBG uptake, its correlation with clinical symptoms in dementia with Lewy bodies. Psychiatry Res 2009; 174: 76-80.

[31] Yoshita M, Taki J, Yokoyama K, et al. Value of ${ }^{123}$ I-MIBG radioactivity in the differential diagnosis of DLB from AD. Neurology 2006; 66: 1850-4.

[32] Novellino F, Bagnato A, Salsone M, et al. Myocardial ${ }^{123}$ I-MIBG scintigraphy for differentiation of Lewy Bodies Disease from FTD. Neurobiol Aging 2009 Jan 8. [Epub ahead of print]

[33] Courbon F, Brefel-Courbon C, Thalamas C, et al. Cardiac MIBG Scintigraphy is a sensitive tool for detecting cardiac sympathetic denervation in Parkinson's Disease. Mov Disord 2003; 18: 890-7.

[34] Taki J, Nakajima K, Hwang EH, et al. Peripheral sympathetic dysfunction in patients with Parkinson's disease without autonomic failure is heart selective and disease specific. Eur J Nucl Med 2000; 27: 566-73.

[35] Reinhardt MJ, Jüngling FD, Krause TM, Braune S. Scintigraphic differentiation between two forms of primary dysautonomia early after onset of autonomic dysfunction: value of cardiac and pulmonary iodine-123 MIBG uptake. Eur J Nucl Med 2000; 27: 595-600.

[36] Orimo S, Ozawa E, Nakade S, Sugimoto T, Mizusawa H. ${ }^{123} \mathrm{I}-$ metaiodobenzylguanidine myocardial scintigraphy in Parkinson disease. J Neurol Neurosurg Psychiatry 1999; 67: 189-94.

[37] Taki J, Yoshita M, Yamada M, Tonami N. Significance of ${ }^{123} \mathrm{I}-$ MIBG scintigraphy as a pathophysiological indicator in the assessment of Parkinson's disease and related disorders: It can be a specific marker for Lewy body disease. Annals of Nucl Med 2004; 18: 453-61.

[38] Barber PA, Varma AR, Lloyd JJ, Haworth B, Snowden JS, Neary D. The electroencephalogram in dementia with Lewy bodies. Acta Neurol Scand 2000; 101: 53-6.

[39] Bonanni L, Thomas A, Tiraboschi P, et al. EEG comparisons in early Alzheimer's disease, dementia with Lewy bodies and Parkinson's disease with dementia patients with a 2-year follow-up. Brain 2008; 131: 609-705.

[40] Tschampa HJ, Neumann M, Zerr I, et al. Decreased CSF amyloid beta42 and normal tau levels in dementia with Lewy bodies. Neurology 2001; 56: 576.
[41] Kanemaru K, Kameda N, Yamanouchi H. Decreased CSF amyloid beta42 and normal tau levels in dementia with Lewy bodies. Neurology 2000; 54: 1875-6.

[42] Parnetti L, Tiraboschi P, Lanari A, et al. Cerebrospinal fluid biomarkers in Parkinson's disease with dementia and dementia with Lewy bodies. Biol Psychiatry 2008; 64: 850-5.

[43] Burton EJ, Barber R, Mukaetova-Ladinska EB, et al. Medial temporal lobe atrophy on MRI differentiates Alzheimer's disease from dementia with Lewy bodies and vascular cognitive impairment: a prospective study with pathologic verification of diagnosis. Brain 2009; 132: 195-203.

[44] Lobotesis K, Fenwick JD, Phipps A, et al. Occipital hypoperfusion on SPECT in dementia with Lewy bodies but not AD. Neurology 2001; 56: 643-9.

[45] Minoshima S, Foster NL, Sima AA, Frey KA, Albin RL, Kuhl DE. Alzheimer's disease versus dementia with Lewy bodies: cerebral metabolic distinction with autopsy confirmation. Ann Neurol 2001; 50: 358-65.

[46] Inui $\mathrm{Y}$, Toyama $\mathrm{H}$, Manabe $\mathrm{Y}$, et al. Evaluation of probable or possible dementia with Lewy bodies using ${ }^{123}$ I-IMP brain perfusion SPECT, ${ }^{123} \mathrm{I}-\mathrm{MIBG}$, and ${ }^{99 \mathrm{~m}}$ Tc-MIBI myocardial SPECT. J Nucl Med 2007; 48: 1641-50.

[47] Tateno M, Kobayashi S, Shirasaka T, et al. Comparison of the usefulness of brain perfusion SPECT and MIBG myocardia scintigraphy for the diagnosis of dementia with Lewy bodies. Dement Geriatr Cogn Disord 2008; 26: 453-7.

[48] Tiraboschi P, Salmon DP, Hansen LA, Hofstetter RC, Thal LJ, Corey-Bloom J. What best differentiate Lewy body from Alzheimer's disease in early-stage dementia? Brain 2006; 129: 729-35.

[49] McKeith IG, Ballard CG, Perry RH, et al. Prospective validation of consensus criteria for the diagnosis of dementia with Lewy bodies. Neurology 2000; 54: 1050-8.

[50] Fujishiro H, Ferman TJ, Boeve BF, et al. Validation of the neuropathologic criteria of the third consortium for dementia with Lewy bodies for prospectively diagnosed cases. J Neuropathol Exp Neurol 2008; 67: 649-56.

[51] O'Brien JT, McKeith IG, Walker Z, et al. Diagnostic accuracy of ${ }^{123}$ I-FP-CIT SPECT in possible dementia with Lewy bodies. Br J Psychiatry 2009; 194: 34-39.

[52] Calvini P, Rodriguez G, Inguglia F, Mignone A, Guerra UP, Nobili F. The basal ganglia matching tools package for striatal uptake semi-quantification: description and validation. Eur J Nucl Med Mol Imaging 2007; 34: 1240-53.

This is an open access article licensed under the terms of the Creative Commons Attribution Non-Commercial License (http: //creativecommons.org/licenses/by-nc/ $3.0 /$ ) which permits unrestricted, non-commercial use, distribution and reproduction in any medium, provided the work is properly cited 